\title{
Glutaraldehyde Crosslinked Polyvinyl Alcohol/Eggshell Powder Biocomposite Films: Properties and Biodegradability
}

\author{
Nur Fazreen Alias and Hanafi Ismail* \\ School of Materials and Mineral Resources Engineering, Universiti Sains Malaysia, \\ Engineering Campus, 14300 Nibong Tebal, Pulau Pinang, Malaysia \\ "Corresponding author: ihanafi@usm.my
}

Published online: 25 August 2020

To cite this article: Alias, N. F. \& Ismail, H. (2020). Glutaraldehyde crosslinked polyvinyl alcohol/eggshell powder biocomposite films: Properties and biodegradability. J. Phys. Sci., 31(2), 45-60. https://doi.org/10.21315/jps2020.31.2.4

To link to this article: https://doi.org/10.21315/jps2020.31.2.4

\begin{abstract}
The utilisation of waste materials in polymer matrices is gaining attraction due to the growing environmental awareness. In this work, eggshells waste was added into polyvinyl alcohol to produce biocomposite films via solution casting method. In order to enhance the properties of the biocomposite, glutaraldehyde (GA) was incorporated as the crosslinking agent. The effects of GA content on the biocomposite were evaluated in terms of its tensile properties, physical properties and biodegradability. From the tensile test, the optimum GA content was found to be 2 phr as portrayed by the highest tensile strength at this GA content. Further addition of GA deteriorated the mechanical properties of the biocomposite. On the other hand, the water absorption, water vapour transmissibility and biodegradability (via natural weathering and soil burial) of the biocomposite films decreased with GA addition.
\end{abstract}

Keywords: Polyvinyl alcohol, eggshell powder, biocomposite, solution casting, glutaraldehyde

\section{INTRODUCTION}

The application of polymer composite materials has been practiced for centuries in almost every field such as electronic, automotive, medical and packaging industries. The growing awareness towards the environmental impact of polymer usage has led to the research and industrialisation of biocomposites. Mass production of polymeric materials is associated to the depletion of fossil fuels, pollutions and persistent polymer degradation. A composite material made up of at least one 
renewable or natural origin component is classified as a biocomposite. ${ }^{1}$ Polymer matrices, either synthetic or bio-based (from plants or animals), combine with natural fibres or inorganic fibres to form biocomposites. A biopolymer is considered as one of the environmentally friendly polymers owing to its biodegradable quality. Moreover, a biodegradable polymer is prone to natural decomposition from microorganism activities, hydrolysis and photodegradation. ${ }^{2}$ Polyvinyl alcohol (PVA) is a synthetic biodegradable polymer, which is attributed by the presence of hydroxyl group in the backbone, making it vulnerable to hydrolysis. PVA is widely used in fibres, textiles, coatings, adhesives and biomedical industry.

Despite being biodegradable, PVA still demonstrates good mechanical and acceptable barrier properties, depending on the degree of hydrolysis and its molecular weight. ${ }^{3}$ Recent literature works revealed that PVA has been widely incorporated in composites and blended with various polymers and additives in order to tailor its properties accordingly to specific applications. Chin et al. had reported on the extraction of microcrystalline cellulose from rice straw via cyclic alkaline and bleaching treatment, and its incorporation in PVA biocomposite films through solution casting process. ${ }^{4}$ The tensile strength and young modulus of the films improved up to $7.5 \mathrm{wt} \%$ with the microcrystalline cellulose addition, attributed to the high surface area of filler, uniform dispersion and good interfacial adhesion.

A study on the mechanical properties of PVA composites by Kumar et al. displayed the tensile properties and moisture uptakes of three different composites: PVA with untreated kenaf, PVA with treated kenaf and PVA with starch. ${ }^{5}$ The highest tensile strength was demonstrated by the PVA/starch composite, which also exhibited a lower moisture uptake than the PVA/kenaf composite. Gaaz et al. reviewed the properties and applications of PVA and halloysite nanotubes (HNT), as well as their nanocomposites. ${ }^{6}$ The PVA/HNT composites were fabricated via electrospinning using a twin-screw extruder and two roll mills. The biocompatibility, non-toxicity and flexibility demonstrated by the composites elevate their potential in biomedical applications. Regarding the mechanical properties of the composites, the tensile strength decreased but the young modulus increased with increasing HNT ratio. Besides, the thermal properties of the PVA was also enhanced by the HNT addition. Meanwhile, Alias et al. worked on the development of new biocomposite based on PVA and palm kernel shell powder applications. ${ }^{7}$ The incorporation of palm kernel shell powder deteriorated the tensile strength and elongation at break of the biocomposite. However, its water absorption and biodegradability properties were enhanced owing to the hydrophilic nature of the natural filler. ${ }^{7}$ 
Nonetheless, one of the drawbacks of composites with natural fibres and waste materials incorporation is the loss of the composites tensile strength. To address the deficiency, various additives may be added. Crosslinking agents such as glutaraldehyde (GA) and silane are added in PVA and its composites to introduce crosslinking within the matrix, resulting in improved mechanical and barrier properties. ${ }^{8}$ Deneke et al. investigated the application of GA as an alternative crosslinking agent in PVA (containing bovine albumin serum) hydrogel membranes via ultraviolet irradiation method. The GA-crosslinked membranes demonstrated excellent performance owing to their ability to retain structural integrity in released buffer as well as consistent thermal degradation. ${ }^{9}$ On the other hand, Hussein et al. studied the effects of crosslinking agent ration and temperature on the degree of swelling in polymer hydrogels by mixing graft polymer of PVA and urea with hydroxypropyl methylcellulose and GA. With increasing GA content, the swelling ratio decreased. Moreover, it was found that the increasing crosslink density in composites has also resulted in higher melting temperature. ${ }^{10}$

To the best of the authors' knowledge, no research works have been reporting on the incorporation of GA as a crosslinking agent in PVA/eggshell powder (ESP) biocomposites. Thus, in this work, the application of GA crosslinking agent in PVA/ESP biocomposite was investigated based on the tensile, physical and biodegradation properties of the biocomposite. This study focuses to improve the strength of the biocomposite without compromising its biodegradability.

\section{EXPERIMENTAL}

\subsection{Materials}

PVA with a density of $1.3 \mathrm{~g} \mathrm{~cm}^{-3}$ and average molecular weight of $89000 \mathrm{~g} \mathrm{~mol}^{-1}$ to $98000 \mathrm{~g} \mathrm{~mol}^{-1}$, and hexamethylenetetramine (HTMA) with molecular weight of $140.19 \mathrm{~g} \mathrm{~mol}^{-1}$ were supplied by Sigma-Aldrich (M) Sdn. Bhd. (Kuala Lumpur, Malaysia). Meanwhile, chicken eggshells were collected at a canteen in the Universiti Sains Malaysia (USM), Penang, Malaysia. GA, glycerol (density of $1.25 \mathrm{~g} \mathrm{~cm}^{-3}$ ), and Tween 80 were purchased from Merck Sdn. Bhd. (Petaling Jaya, Malaysia). GA and HTMA were used as the crosslinking agent and reagent respectively in this study. Meanwhile, glycerol functioned as a plasticiser in the composite to improve its flexibility and aid in the processing. Tween 80 on the other hand acted as a deformer and paraffin wax to provide smooth film surface. Deionised water was used as the solvent for solution casting. Generally, all chemicals involved were of analytical grade. 


\subsection{Preparation of Biocomposite Films}

ESP was prepared by cleaning chicken eggshells thoroughly with tap water, followed by drying at room temperature for $24 \mathrm{~h}$. Grinding process was then carried out by using a mini crusher (Model C03-43B0, Chyun Tseh Industrial Co. Ltd, Taichung, Taiwan) to produce ESP. The ESP was sieved using a sieving plate of $75 \mu \mathrm{m}$ mesh size, dried at $70^{\circ} \mathrm{C}$ for $24 \mathrm{~h}$, and kept in a desiccator for further use. The biocomposite films were prepared via solution casting method. PVA was firstly added into a beaker of $200 \mathrm{ml}$ deionised water and the mixture was stirred for $10 \mathrm{~min}$ at $95^{\circ} \mathrm{C}$. Subsequently, ESP was added and the stirring was continued for $10 \mathrm{~min}$. Additives were then added and the mixture was stirred for another $10 \mathrm{~min}$. At the end of the process, the solution was cast over a glass plate and left to dry for overnight at room temperature, forming biocomposite film. The film was further dried in an oven at $70^{\circ} \mathrm{C}$ for $2 \mathrm{~h}$, before being peeled off and kept in a desiccator until further characterisation was conducted. PVA/ ESP biocomposites were prepared using variable GA contents with fixed weight percentage of PVA/ESP at $80 / 20 \mathrm{wt} \%$. The biocomposite films formulation used is as shown in Table 1.

Table 1: Formulation of biocomposite films.

\begin{tabular}{|c|c|c|c|c|c|c|c|}
\hline \multirow[b]{2}{*}{ Composition } & \multirow{2}{*}{$\begin{array}{c}\text { PVA } \\
\text { Actual } \\
\text { weight (g) }\end{array}$} & \multirow{2}{*}{$\begin{array}{c}\text { ESP } \\
\text { Actual } \\
\text { weight }(\mathrm{g})\end{array}$} & \multicolumn{2}{|r|}{ GA } & \multirow{2}{*}{$\begin{array}{c}\text { Glycerol } \\
\text { Actual } \\
\text { weight }(\mathrm{g})\end{array}$} & \multirow{2}{*}{$\begin{array}{c}\text { Tween } 80 \\
\text { Actual } \\
\text { weight }(\mathrm{g})\end{array}$} & \multirow{2}{*}{$\begin{array}{c}\text { HTMA } \\
\text { Actual } \\
\text { weight }(\mathrm{g})\end{array}$} \\
\hline & & & $\mathrm{phr}$ & $\begin{array}{c}\text { Actual } \\
\text { weight }(\mathrm{g})\end{array}$ & & & \\
\hline $80 / 20 / 0$ & 8 & 2 & 0 & 0.0 & 1.5 & 0.1 & 0.6 \\
\hline $80 / 20 / 1$ & 8 & 2 & 1 & 0.1 & 1.5 & 0.1 & 0.6 \\
\hline $80 / 10 / 2$ & 8 & 2 & 2 & 0.2 & 1.5 & 0.1 & 0.6 \\
\hline $80 / 20 / 3$ & 8 & 2 & 3 & 0.3 & 1.5 & 0.1 & 0.6 \\
\hline $80 / 20 / 4$ & 8 & 2 & 4 & 0.4 & 1.5 & 0.1 & 0.6 \\
\hline
\end{tabular}

\subsection{Characterisation of Biocomposite Films}

\subsubsection{Tensile test}

The tensile properties of the biocomposite films were determined using Instron 3366 testing machine (Petaling Jaya, Malaysia), operated at crosshead speed of $50 \mathrm{~mm} \mathrm{~min}{ }^{-1}$ and load cell of $10 \mathrm{kN}$ at room temperature. The gauge length was set at $50 \mathrm{~mm}$. Prior to testing, the biocomposite films were cut into dumbbell-shaped specimens (ASTM D638) and their thickness was measured. An average tensile strength from five repeated tests was recorded for each composite composition. 


\subsubsection{Surface morphological study}

Prior to morphological observations, the specimens were mounted on aluminium stubs and sputter-coated with thin gold layer in order to prevent electrostatic charging and poor resolution. The surface of each biocomposite film was observed by using a field emission scanning electron microscope (FESEM) (Model Zeiss Supra 35VP) (Oberkochen, Germany).

\subsubsection{FTIR analysis}

The functional groups present in the specimens were evaluated via Perkin Elmer Fourier transform infrared (FTIR) spectroscopy (Boston, United States) equipped with attenuated total reflectance (ATR) mode in the range of $400-4000 \mathrm{~cm}^{-1}$. The mean peaks obtained within 16 times scanning were used for further analysis.

\subsubsection{Water absorption test}

Water absorption test (ASTM D570) was carried out by immersing the specimens in distilled water at room temperature. After $24 \mathrm{~h}$, the water adhering on the specimen surface was removed and the weights of the specimens were measured. The water absorption percentage was calculated as:

$$
\mathrm{W}_{\mathrm{A}}(\%)=\left[\left(\mathrm{W}_{\mathrm{f}}-\mathrm{W}_{\mathrm{i}}\right) / \mathrm{W}_{\mathrm{i}}\right] \times 100
$$

where $\mathrm{W}_{\mathrm{f}}$ is the final specimen weight and $\mathrm{W}_{\mathrm{i}}$ is the initial dry weight of specimen.

\subsubsection{Water vapour transmissibility test}

Water vapour transmissibility test was conducted by sealing the biocomposite film over a container containing $30 \mathrm{ml}$ distilled water. The initial weight of the set-up was recorded before it was placed in a desiccator at room temperature. The setup was weighed daily until a constant weight was achieved. The average value of set-up weight was calculated using Equation 2, representing the WVT of each composite composition.

$$
\mathrm{WVT}=(\mathrm{G} / \mathrm{t}) / \mathrm{A}
$$

where $\mathrm{G}$ is the weight gain of set-up, $\mathrm{t}$ is the test duration, and $\mathrm{A}$ is the total test area. 


\subsubsection{Biodegradability test via natural weathering and soil burial methods}

The specimens for biodegradability test were cut into dumbbell shape according to ASTM D882 (Type IV) and the initial weights of the specimens were recorded. For the natural weathering test, the specimens were exposed to natural weather for 14 days, while for the soil burial test, the specimens were buried in natural soil for 4 days. Both tests were conducted at the School of Materials and Mineral Resources Engineering, USM. The final weights of all specimens after the biodegradability tests were recorded and the changes in weight were determined.

\section{RESULTS AND DISCUSSION}

\subsection{Tensile Properties}

Figure 1 displays the tensile strength results for the PVA/ESP biocomposite with different GA compositions. It was found that the highest tensile strength was achieved at $2 \mathrm{phr}$ GA content incorporation into the biocomposite. The tensile strength of the biocomposite decreased as higher amount of GA was added. Theoretically, when a low content of GA was added, crosslinks may not be formed at a high degree as some of the aldehyde groups in GA are not reacted. At the optimum GA content of $2 \mathrm{phr}$, the formation of crosslinks is promoted at both aldehyde sides. However, when the GA content was increased to beyond $2 \mathrm{phr}$, there is a tendency of over-crosslinking that may result in the deterioration of the mechanical properties of the biocomposite. Similar finding was reported by Ismail and Zaaba for sago starch crosslinked plastic films. ${ }^{11}$

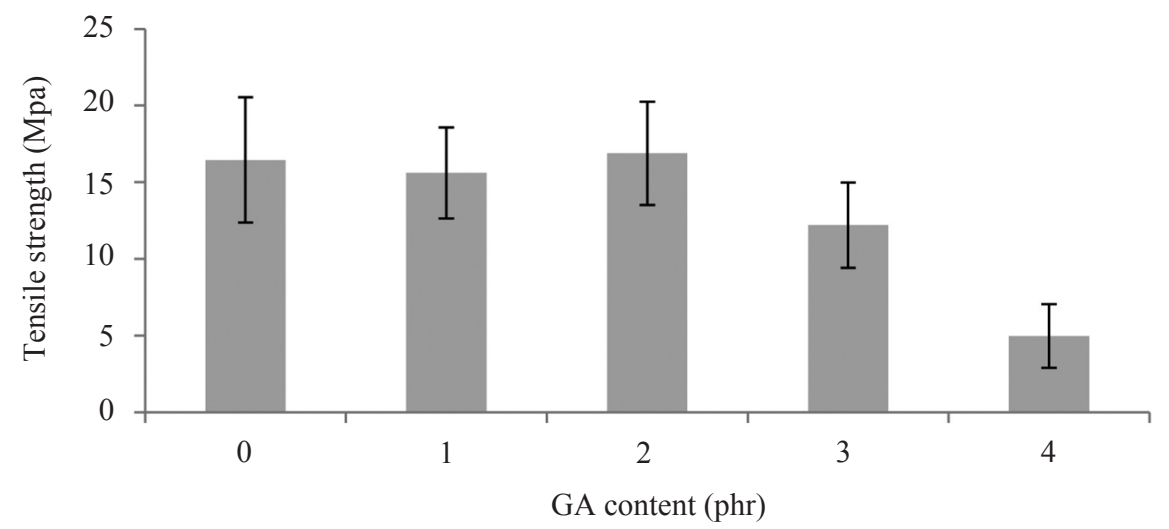

Figure 1: Tensile strength of PVA/ESP biocomposites with different GA contents. 
The effect of varying GA contents addition on the elongation at break of the PVA/ESP biocomposite is portrayed in Figure 2. The elongation at break of the biocomposite decreased as the composition of GA was increased. The tensile strength of the film with $0.1 \mathrm{~g}$ GA was lower than those of the neat PVA and film with $2 \mathrm{phr}$ GA. This is probably due to the insufficient addition of crosslinking agent in the biocomposite, resulting in slightly lesser interaction between GA and the polymer chains. Nonetheless, when 2 phr of GA was added, the GA is able to adequately crosslink the biocomposite and thus improves its strength. Excessive crosslinking agent content in a composite however may lead to over-crosslinking.

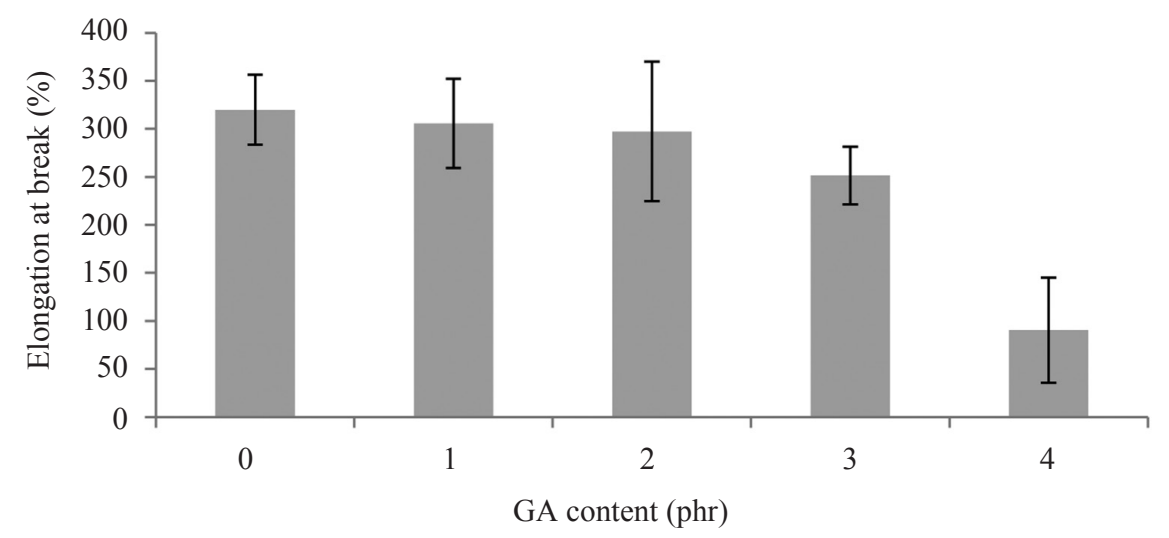

Figure 2: Elongation at break of PVA/ESP biocomposites with different GA contents.

Figure 3 presents the effect of GA incorporation into PVA/ESP biocomposite on its tensile modulus. An increase in the GA content up to $2 \mathrm{phr}$ caused an increment in the tensile modulus of the PVA/ESP biocomposite. Beyond $2 \mathrm{phr}$ GA content, the tensile modulus of the film decreased drastically. For the control sample without GA addition, its tensile modulus was slightly lower than the film with the optimum GA content. This is due to the absence of crosslinking structure that results in weaker intermolecular forces.

Tensile modulus measures the resistance of a material to deformation. A high tensile modulus was obtained at $2 \mathrm{phr}$ GA content which is due to the formation of crosslinks between the polymer chains. The crosslinks present in the structure will prevent the molecules from sliding past each other under deformation, which makes it stiffer. The decrement in the properties can be explained as a result of competitive action among three factors during crosslinking. These factors include the introduction of flexibility by moieties, diminution of existing physical network, and formation of chemical structure. ${ }^{12}$ 


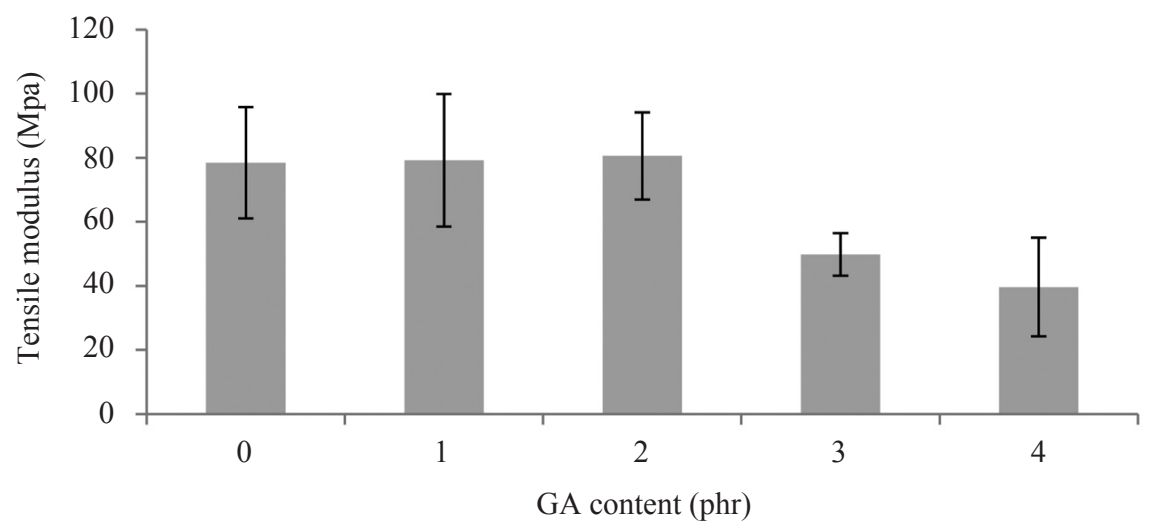

Figure 3: Tensile modulus of PVA/ESP biocomposites with different GA content.

\subsection{Surface Morphological Study}

Scanning electron microscopy (SEM) was employed to study the surface morphology of the PVA/ESP films with GA crosslinking agent. Figure 4(b) illustrates the surface morphology of the PVA/ESP film with $2 \mathrm{phr}$ GA content. From the micrograph, slight agglomeration of ESP and formation of bubbles on the films as a result of air entrapment during the casting process were observed. However, the filler is considered to be well-dispersed and the white spots are assumed to be the eggshell powder. Consequently, the film demonstrated better tensile properties as reported earlier.

In comparison, the biocomposite film with $2 \mathrm{phr}$ GA exhibited smooth surface structure while with the addition of $4 \mathrm{phr}$ GA, rough surfaced PVA/ESP biocomposite film was produced. The dispersion of filler becomes poor as agglomeration occurs, causing roughness on the film surface. The SEM micrographs also showed correlation to the tensile properties of the films. Due to agglomeration, the stress transfer between matrix and filler becomes poor, causing a drop in the tensile properties. The micrographs proved that the addition of lesser amount of GA in the film provided better filler dispersion and interaction between GA and PVA. High GA content may lead to the diminution of the existing filler network due to the formation of hydrogen bonding between the aldehyde groups in GA and the polymer. 

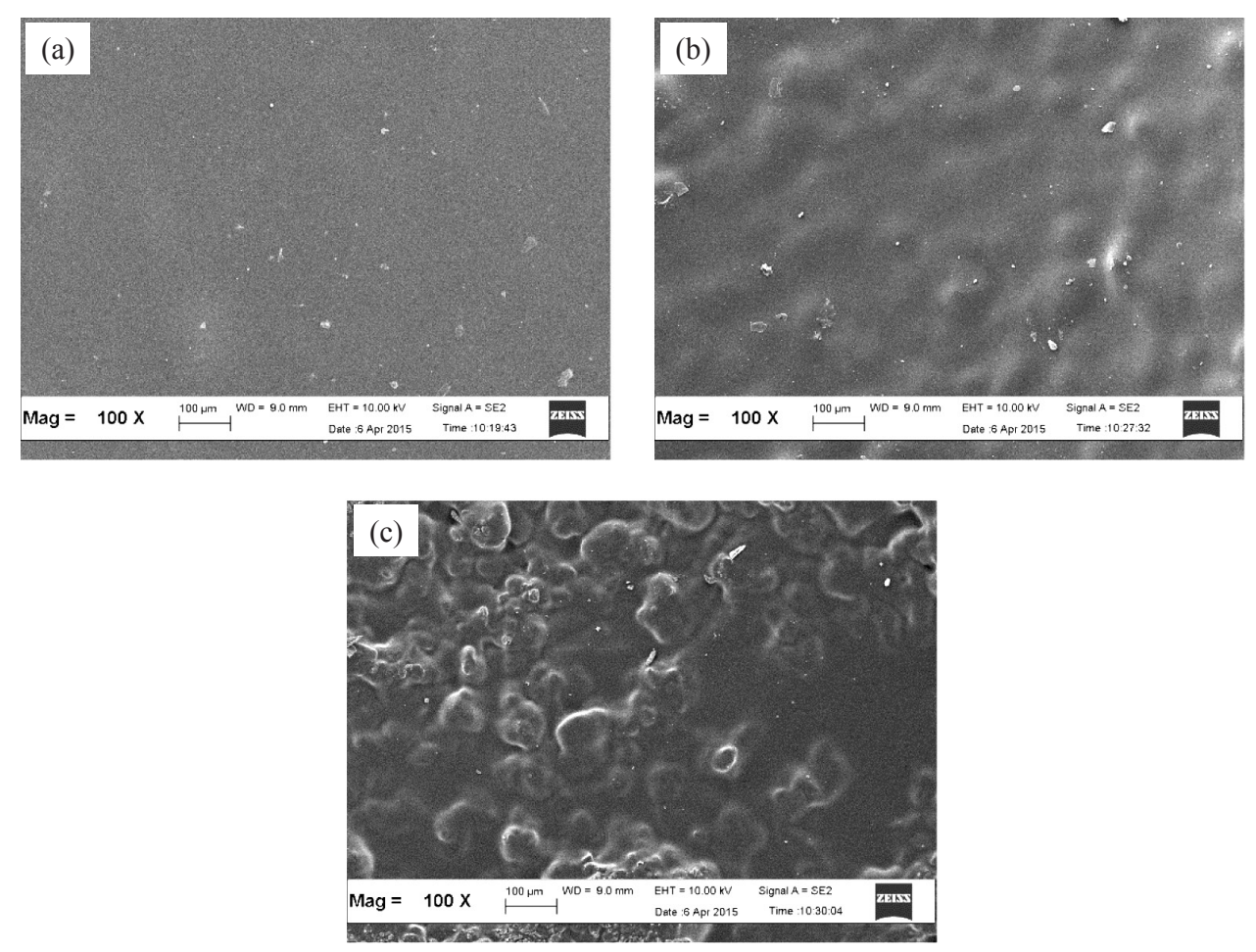

Figure 4: SEM micrographs of PVA/ESP biocomposites showing (a) without GA, (b) with 2 phr GA, and (c) with 4 phr GA.

\subsection{Chemical Composition by FTIR}

Figure 5 illustrates the FTIR spectra of the GA incorporated PVA/ESP biocomposite films. The broad band in the $3600-3100 \mathrm{~cm}^{-1}$ region, which is due to the $\mathrm{OH}$-stretching vibration in PVA, provided information concerning the presence of hydrogen bond in the films. Besides that, the FTIR absorption band around $1400 \mathrm{~cm}^{-1}$ was assigned to the symmetric $\mathrm{CH}_{2}$ bending vibration.

The absorption band at $2942.71 \mathrm{~cm}^{-1}$ was associated with the alkane group, represented by the $\mathrm{C}-\mathrm{H}$ bonds stretch. The intensity of this band was the highest in the PVA/ESP film with $2 \mathrm{phr}$ GA. This band is known as the "crystallinity band", where an increase in the band intensity reflects increment in the degree of crystallinity of the samples. ${ }^{13}$ Therefore, it is proven that the addition of $2 \mathrm{phr}$ GA provides a favourable impact on the crosslinking of the polymer chains, thus forming higher crystallinity biocomposite. 


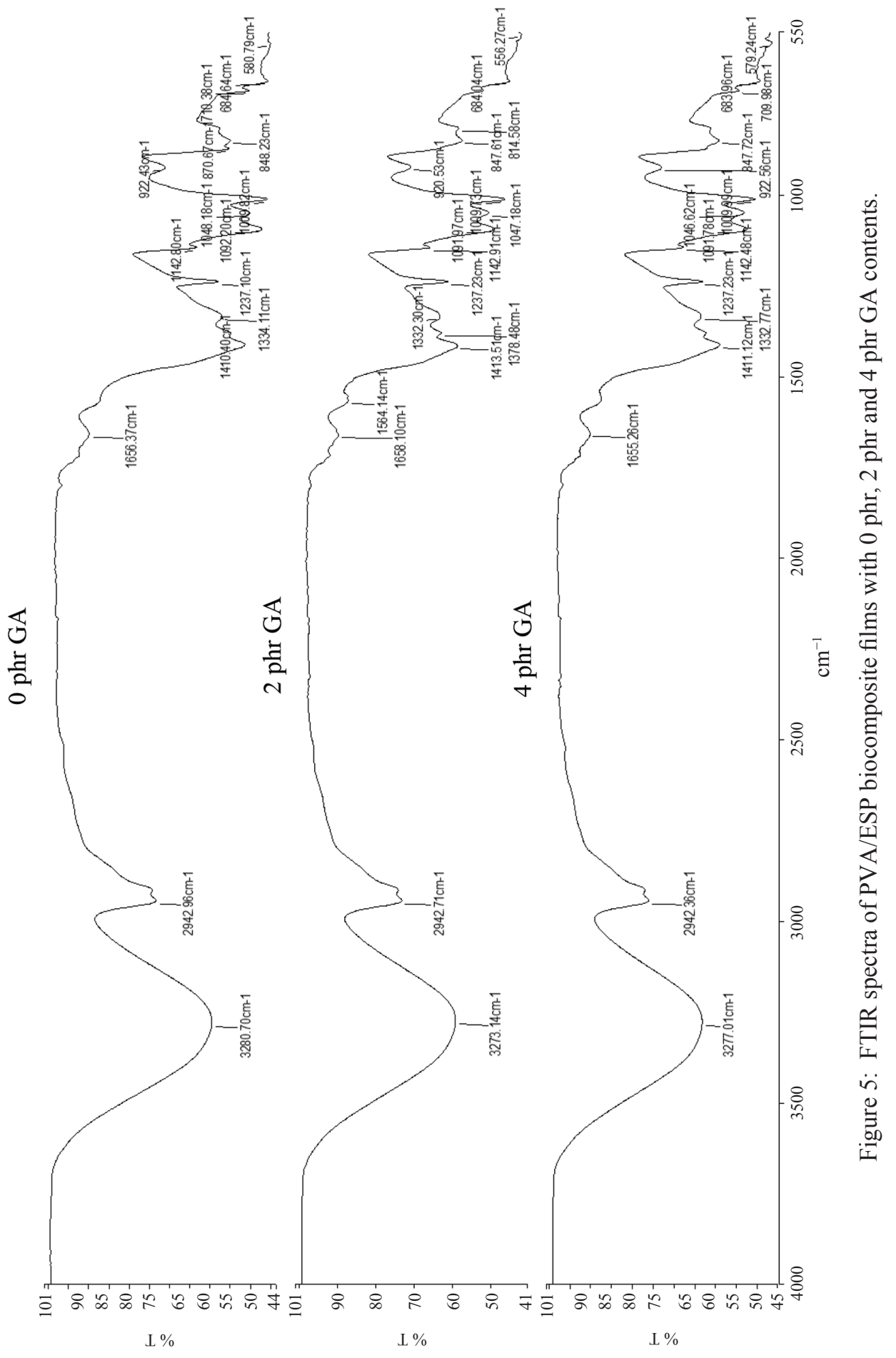


Comparing these three FTIR spectra, there are some different peaks that can be observed. For instance, in the spectrum of PVA/ESP film with 2 phr GA, an additional peak at $1564 \mathrm{~cm}^{-1}$ was observed, which may indicate the presence of aldehyde in the GA. Typically, the carbonyl stretch, $\mathrm{C}=\mathrm{O}$ of saturated aliphatic aldehydes appears within the $1740-1720 \mathrm{~cm}^{-1}$ absorption bands. The shift in the vibration peak might be due to the presence of unsaturated carbons adjacent to the aldehyde group, which causes the peak to shift to a lower wavenumber.

\subsection{Water Absorption}

Figure 6 shows the variation in the percentage of water absorbance of the PVA/ ESP biocomposite films, where the water absorption decreased upon the addition of GA. The water absorption was the highest in the control sample due to the fact that no cross-linking agent was present to counter the water soluble characteristic of PVA, similarly as reported in an earlier work on the water absorption properties of kenaf fibre/PVA composites. ${ }^{14}$ The hydroxyl group is hydrophilic in nature which contributes to the absorption of high amount of water into the film.

Only a slight drop of water absorbance was observed with the addition of GA up to $1 \mathrm{phr}$. The decrement in water absorption was more noticeable with the addition of $2 \mathrm{phr}$ GA. The reduction is due to the crosslinking effect of GA and the polymer itself which allowed lesser water to penetrate into the film. This was further attributed to the formation of network structures by the crosslinking agent, which improved water resistance and prevented water molecules from dissolving the biocomposite films.

With the incorporation of GA, the immobilised phases increased and the inclusion of water into the polymer will be difficult. Beyond $2 \mathrm{phr}$ GA content, the water absorption of the films increased. This is due to the network formed and also the effectiveness of GA at higher contents than optimum. In general, the addition of GA in biocomposites helps to reduce water uptake into them. According to Ismail and Zaaba, the heterogeneous crosslinking formed by GA tends to produce more hydrophobic structure, in which the network structure prevents water molecules from dissolving the films and at the same time improves the water resistance of the films. ${ }^{15}$ 


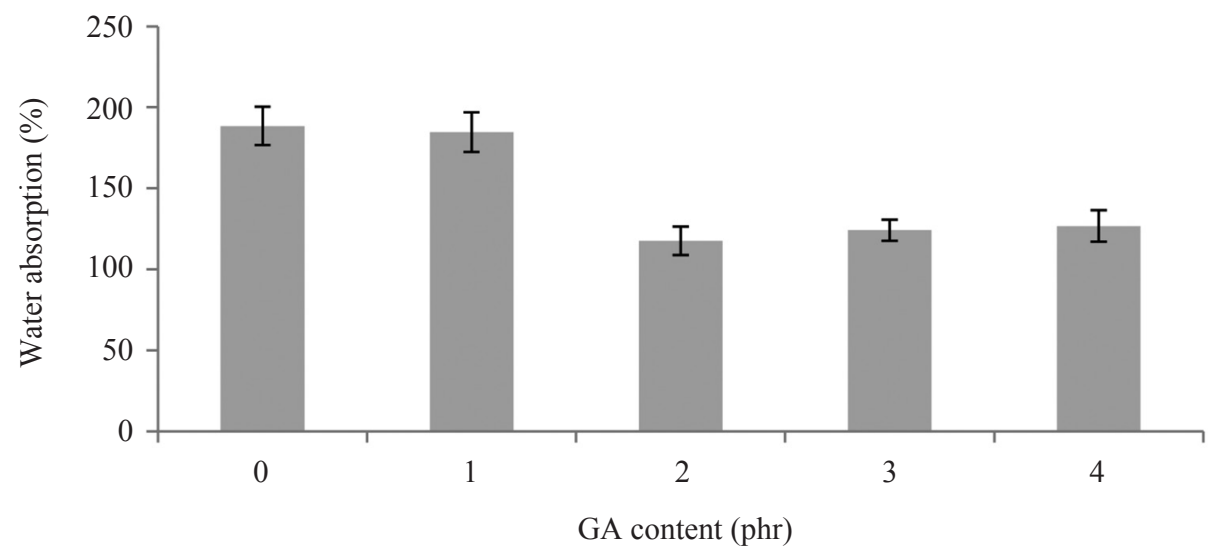

Figure 6: Water absorption percentage of PVA/ESP biocomposites with different GA contents.

\subsection{Water Vapour Transmission}

Water vapour transmission (WVT) is generally applied to measure the capability of water vapour to penetrate through a material. Figure 7 presents the water vapour transmission of the PVA/ESP biocomposites with different GA contents. It is shown that the addition of GA varied the WVT of the PVA/ESP films. Initially, as GA was added, the WVT decreased. However, when $3 \mathrm{phr}$ and more GA was added, the WVT started to increase significantly.

The crosslinking between the polymer chains tends to reduce the intermolecular space between them, therefore resulting in reduction in the water vapour permeable values. ${ }^{16}$ The incorporation of GA improved the interaction between molecules, thus improving the cohesiveness of a biopolymer matrix and decreasing its water sensitivity. ${ }^{17}$ For the PVA/ESP biocomposite film with 2 phr GA, the reduction of the water permeability originated from the increased path tortuosity of the penetrant molecules (water molecules) and also the enhanced modulus of the polymer matrix in the composite films. ${ }^{18}$ 


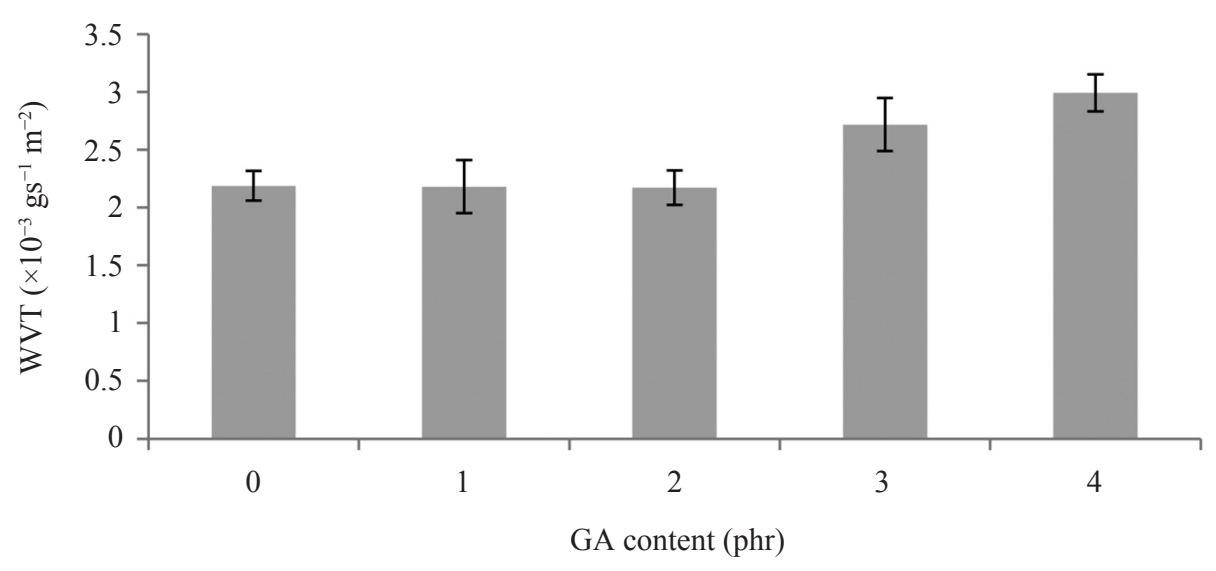

Figure 7: WVT of PVA/ESP films with different GA contents.

\subsection{Biodegradability via Natural Weathering and Soil Burial}

Figure 8 displays the weight loss after weathering of the PVA/ESP biocomposites with different GA crosslinking agent contents. The weight loss varied according to the GA content. The highest weight loss was exhibited by the biocomposite without GA due to the absence of crosslink in the structure, causing the degradation to occur easier than the films with crosslinked structure.

The lowest weight loss was exhibited by the film with the addition of $2 \mathrm{phr}$ GA, which is considered as the optimum amount of crosslinking agent. This implies that with this amount of GA, better crosslink formation occurs, which makes the film more resistant to degradation. Beyond $2 \mathrm{phr}$ GA addition, the weight loss increased slightly, which might be due to the ineffective crosslink structures as they are generally saturated or over-crosslinked.

In a soil burial or composting test, the degradation of PVA occurs via strong microbial activity on the surface of the specimen. By-products such as carbon dioxide, biomass and soluble organic compounds are produced through polymeric degradation. ${ }^{19}$ Kale et al. reported that PVA is a biodegradable material that undergoes reduction in its molecular weight by hydrolysis depolymerisation. ${ }^{20} \mathrm{An}$ important factor that influences the degradability of a polymer is its hydrophilicity, where an increase in hydrophilicity facilitates biodegradation. While buried in soil, water will diffuse into the films, causing swelling and therefore enhancing biodegradation. 


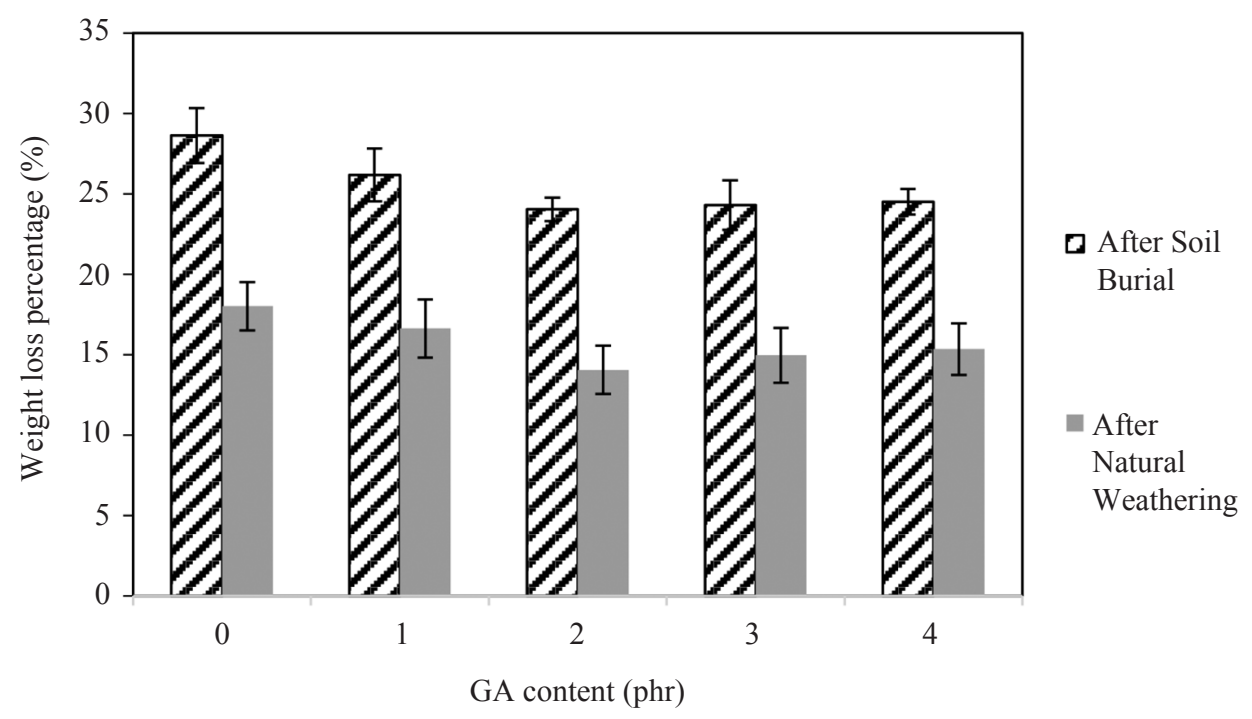

Figure 8: Weight loss after soil burial of PVA/ESP biocomposites with different GA contents.

The weight loss trends for both soil burial and natural weathering tests were identical. With $2 \mathrm{phr}$ GA content, smaller weight loss was observed in the films, indicating the optimum crosslinking agent content. However, in natural weathering, degradation occurs via photo-degradation from the absorption of ultraviolet radiation. This eventually induces chain scission and reduction in molecular weight within the polymer matrix as well as leaching of fillers. ${ }^{21}$

The main factor in consideration is the effect of crosslink networks formed by GA on the biocomposite films degradation. From the graph in Figure 8, the lowest weight loss was demonstrated by the film with the addition of $2 \mathrm{phr}$ GA, herein proved the optimum crosslink formation in the PVA film. Besides that, the crosslink structure reduced the water penetration into the film, which may slow down the degradation process.

\section{CONCLUSION}

The tensile properties of the biocomposite films of study increased with increasing GA content up to $2 \mathrm{phr}$, but drastically dropped with higher amount of GA addition. GA addition therefore could enhance the tensile properties of PVA/ ESP film. The morphological studies on the films surface indicated that the filler was well dispersed throughout the matrix with slight agglomeration when $2 \mathrm{phr}$ GA was incorporated. On the other hand, when more than 2 phr GA was added, 
the filler agglomeration increased, resulting in lower tensile properties. The GA content of $2 \mathrm{phr}$ appeared to be optimum as the tensile properties in tension tends to decrease above this value. The water absorption properties of the films were found to be greatly affected by the addition of the crosslinking agent. PVA/ESP biocomposite film with $2 \mathrm{phr}$ GA exhibited the lowest water absorption among the other films. This is because of the formation of crosslink networks by GA, which reduces the free volume and prevents water to penetrate into the film. For biodegradability properties, it was found that the addition of crosslinking agent reduced the biodegradability of the biocomposite films. The crosslinks formed have enhanced the network structure of the film, causing it to be less prone to degradation either by weathering or soil burial.

\section{ACKNOWLEDGEMENTS}

The authors would like to thank the School of Materials and Mineral Resources Engineering, USM for providing necessary facilities for the accomplishment of this research. Great appreciation is conveyed to those who have been directly or indirectly involved in this work.

\section{REFERENCES}

1. Ahmed, S. et al. (2018). Biocomposites: Biomedical and environmental applications. Florida: CRC Press.

2. Jain, N., Singh, V. K. \& Chauhan, S. (2018). A review on mechanical and water absorption properties of polyvinyl alcohol based composites/films. J. Mech. Behav. Mat., 1-9. https://doi.org/10.1515/jmbm-2017-0027

3. Aslam, M., Kaylar, M. A. \& Raza, Z. A. (2018). Polyvinyl alcohol: A review of research status and use of polyvinyl alcohol based nanocomposites. Polym. Eng. Sci., 1-14. https://doi.org/10.1002/pen.24855

4. Chin, K. et al. (2017). Extraction of microcrystalline cellulose from rice straw and its effect on polyvinyl alcohol biocomposites film. AIP Conf. Proc., 1865, 04006. https://doi.org/10.1063/1.4993348

5. Kumar, J. P., Reddy, P. R. \& Laxminaraya, P. (2018). Mechanical properties of polyvinyl alcohol composites. Int. J. Eng. Tech. Res., 5(4), 1051-1062.

6. Gaaz, T. S. et al. (2015). Properties and applications of polyvinyl alcohol halloysite nanotubes and their nanocomposites. Mol., 20(12), 22833-22847. https://doi.org/10.3390/molecules201219884

7. Alias, N. F. et al. (2018). Development of new material based on polyvinyl alcohol/ palm kernel shell powder biocomposites. Adv. Env. Studies, 2(2), 98-107.

8. Aisverya, S. et al. (2018). Preparation and characterization of chitosan-povidone blend with glutaraldehyde as crossliking agent. World J. Pharm. Res., 7(5), 1926-1940. https://doi.org/10.20959/wjpr20186-11460 
9. Deneke, N. et al. (2018). Evaluating alternative crosslinking agent in poly (vinyl alcohol) hydrogels membranes. Purs., 1(2), 63-81.

10. Hussein, A. K., Khalil, B. I. \& Abud, H. H. (2017). Effect of crosslinking agent ratio and temperature on degree of swelling in polymer hydrogels. Chem. Process Eng. Res., 52, 1-9.

11. Ismail, H. \& Zaaba, N. F. (2012). Tensile properties, degradation behavior and water absorption of sago starch plastic films. J. Vinyl Additives Tech., 235-246. https://doi.org/ 10.1002/vnl.20300

12. Krumova, M. et al. (2000). Effect of crosslinking on the mechanical and thermal properties of poly(vinyl alcohol). Polym., 41(26), 9265-9272. https://doi.org/10 .1016/S0032-3861(00)00287-1

13. Abdulkhani, A. et al. (2013). Preparation of cellulose/polyvinyl alcohol biocomposite films using 1-N-Butyl-3-methylimidazolium chloride. Int. J. Biol. Macromol., 62, 379-386. https//doi.org/10.1016/j.ijbiomac.2013.08.050

14. Tan, B. K. et al. (2014). Water absorption properties of kenaf fibre - poly (vinyl alcohol) composites. Mat. Res. Innov., 18, 144-146. https://oi.org/10.1179/ 1432891714Z. 000000000946

15. Ismail, H. \& Zaaba, N. F. (2012). The mechanical properties, water resistance and degradation behaviour of silica-filled sago starch/pva plastic films. J. Elast. Plast., 46(1), 96-109. https//doi.org/10.1177/0095244312462163

16. Ooi, Z. X. et al. (2012). Properties of the crosslinked plastized biodegradable poly(vinyl alcohol)/ rambutan skin waste flour blends. J. Appl. Polym. Sci., 125, 1127-1135. https://doi.org/10.1002/app.34860

17. Taghizadeh, M. \& Sabouri, N. (2013). Biodegradation behaviors and water adsorption of poly (vinyl alcohol)/starch/carboxymethyl cellulose/clay nanocomposites. Int. Nano Lett., 3, 51-65. https://doi.org/10.1186/2228-5326 $-3-51$

18. Strawhecker, K. \& Manias, E. (2006). Nanocomposites based on water soluble polymers and unmodified smectite clays. In Yu, Z. Z. \& Mai, Y. W. (Eds). Polymer nanocomposites. New York: CRC Press, 206-233.

19. Mohamed, R. et al. (2018). Effect of thermal characteristics of different plasticizers for pvoh/sago ss blends and its weight loss degradation upon soil burial. AIP Conf. Proc. 1985, 030013. https://doi.org/10.1063/1.5047171

20. Kale, G. et al. (2007). Composability of bioplastic packaging materials: An overview. Macromol. Biosci., 7, 255-277. https://doi.org/10.1002/mabi .200600168

21. Pang, A. L., Ismail, H. \& Abu Bakar, A. (2018). Linear low density polyethylene/ poly vinyl alcohol/ kenaf composites: Effect of natural weathering on functional group, weight loss characteristics, tensile, morphological and thermal properties. Sains Malays., 47(3), 571-580. https//doi.org/10.17576/jsm-2018-4703-18 